\title{
Traveling wave solutions of a nonlinear degenerate parabolic system from petroleum engineering
}

\author{
MiCHIEL BERTSCH ${ }^{\dagger}$ \\ Istituto per le Applicazioni del Calcolo "Mauro Picone”, Consiglio Nazionale delle Ricerche, \\ Viale del Policlinico, 137, I-00161 Roma, Italy, and \\ Dipartimento di Matematica, Università degli Studi di Roma “Tor Vergata”, \\ Via della Ricerca Scientifica, I-00133 Roma, Italy \\ CARLO NitsCH \\ Dipartimento di Matematica e Applicazioni "R. Caccioppoli", \\ Università degli Studi di Napoli "Federico II", \\ Via Cintia, Monte S. Angelo I-80126 Napoli, Italy
}

[Received 7 March 2007 and in revised form 1 January 2008]

\begin{abstract}
We study existence and qualitative properties of traveling wave solutions of a new free boundary problem which describes fluid flow in diatomite rocks. Diatomites are rather fragile and are characterized by low permeability, which can increase due to the nonlocal accumulation of damage caused by the fluid flow. The traveling wave solutions give insight into the behavior near the free boundaries and show a strong parameter dependence. In particular, we find in certain parameter ranges solutions with discontinuities across the free boundaries.
\end{abstract}

Keywords: Traveling waves; nonlinear parabolic system; degenerate parabolic equations; PerronHadamard; center manifold; nonlocal damage mechanics; oil engineering.

\section{Introduction}

In this paper we study traveling wave solutions of the system

$$
\left\{\begin{array}{l}
\omega_{t}=\varepsilon^{2}\left(\omega^{\mu}(p-I)_{+}^{\beta} \omega_{x}\right)_{x}+a(1-\omega)(p-I)_{+}^{\gamma}, \\
p_{t}=\left(\omega^{\alpha} p_{x}\right)_{x},
\end{array}\right.
$$

where $a, \mu, \alpha, \beta, \gamma, \varepsilon$ are positive constants and $I \geqslant 0$. The subscript + indicates the positive part $\left(y_{+}=\max \{y, 0\}\right)$.

System (1) is motivated by a model presented by Barenblatt \& al. [4] for fluid flows in fragile porous rock, for instance in oil bearing diatomite formations. The confined one-dimensional flow of a slightly compressible fluid, in a deep reservoir made of elastic porous rock, is described in the literature [3] by the following equation:

$$
p_{t}=\left(K p_{x}\right)_{x}
$$

†E-mail: m.bertsch@iac.cnr.it

†Corresponding author. E-mail: c.nitsch@unina.it 
Here $p \geqslant 0$ represents the pressure of the fluid and $K$ is a coefficient proportional to the permeability of the rock. Observations in the diatomite oil fields [4] suggest that the permeability of this rock is an increasing function of the number of the accumulated microcracks. The latter physical quantity can be taken into account by including into the equations the well known damage parameter $\omega(x, t)$, which is the fraction of the broken bonds at time $t$ around a point $x$, averaged in a proper way [11]. The damage $\omega$ is by definition a scalar field which takes values between 0 and 1 . The key point in [4] was the assumption that $K=K(\omega)$ with $K(0)=0$ and $K^{\prime}>0$. Furthermore, to close the equations, it was postulated that the damage evolves according to a nonlocal equation (see also [5]) of the form

$$
\omega_{t}=\left[\left(D(\omega, p) \omega_{x}\right)_{x}+f(\omega, p)\right]_{+} .
$$

Here $D$ and $f$ are positive nondecreasing functions of $p$. Both $f$ and $D$ are equal to zero if $p \leqslant I$, where $I$ is a nonnegative constant which is related to the strength of the diatomite rock. Moreover, we can assume that $f$ is some increasing function of the pressure $p$, and proportional to the fraction of unbroken bonds $(1-\omega)$. The subscript + expresses the impossibility of damage healing. Since all traveling wave solutions which we shall construct in this paper satisfy $\omega_{t} \geqslant 0$, we have omitted the subscript + in system (1). In this context (1) can be considered as a model system (see also [2, 11]). Its particular form makes it possible to distinguish several ranges of parameters $\alpha, \beta, \gamma, \mu$ and $I$.

In [6] we have constructed nonnegative compactly supported solutions, $(\omega(x, t), p(x, t))$, of the system of partial differential equations (11). In addition it is shown that the spatial supports of $\omega$ and $p$ coincide,

$$
\operatorname{supp} \omega(t)=\operatorname{supp} p(t) \quad \text { for } t \geqslant 0
$$

(we assume without loss of generality that $p=0$ corresponds to the rest pressure of the fluid in the undamaged zone of the oil reservoir) and that

$$
p>I \quad \text { in the interior of the support for } t \geqslant 0,
$$

assuming that these properties hold at $t=0$. In fact, $\omega$ and $p$ are smooth solutions of the pde's in the interior of their supports. Finally, it has been shown that the supports do not shrink:

$$
\operatorname{supp} \omega\left(t_{1}\right) \subseteq \operatorname{supp} \omega\left(t_{2}\right) \quad \text { if } 0 \leqslant t_{1} \leqslant t_{2} .
$$

According to these results system (1) can be viewed as a free boundary problem, and it is natural to ask how $\omega$ and $p$ behave near the boundary of their support. In [6] it has been shown that the product

$$
\omega(p-I)_{+} \quad \text { vanishes at the interface for almost every } t>0,
$$

but this leaves open the question if $\omega$ and $(p-I)_{+}$can have jumps at the interfaces.

In the present paper we shall construct traveling wave solutions which show that indeed $\omega$ and $(p-I)_{+}$can have jumps, and that their behavior strongly depends on the parameters in the problem. In Section 3 we state and discuss the main results, comparing with some simplified problems described in Section 2 In Section 4 we rewrite the equations for the traveling waves in a more suitable way. In the rest of the paper we prove the results. 


\section{Some preliminary remarks}

Our main results are quite complicated due to a strong parameter dependence. In particular, it turns out that the number of traveling wave solutions depends on the values of the parameters in the system. In this section we shall discuss very briefly two different generalizations of the so-called porous medium equation:

$$
u_{t}=\varepsilon^{2}\left(u^{\mu} u_{x}\right)_{x},
$$

where $\mu>0$. In this way we will obtain, at least at an intuitive level, some insight into how the number of traveling wave solutions can vary for our system (see the discussion at the end of Section 3).

It is well-known that for any positive wave speed, $\lambda>0$, equation 6 has a unique (up to translation) traveling wave solution,

$$
\left(\frac{\lambda \mu}{\varepsilon^{2}}(\lambda t-x)_{+}\right)^{1 / \mu}
$$

and that any general solution of (6) behaves near a moving interface (say with interface velocity $\lambda>0)$ as such a traveling wave solution ([1, 12]).

As a first generalization we consider the equation

$$
u_{t}=\varepsilon^{2}\left(u^{\mu} u_{x}\right)_{x}+u^{q},
$$

where $0<q<1$. The traveling wave solutions of $(8)$ have been extensively studied in the literature; for their complete characterization and additional references we refer to Chapter 7 of the book by Gilding and Kersner [8]. In particular, for all $\lambda>0$ and $\mu>0$ there exists a unique traveling wave solution in a neighborhood of $x=\lambda t$ which behaves like (7). On the other hand, if $\varepsilon=0$ there exists exactly one nontrivial traveling wave solution

$$
u_{q}(x, t):=\left(\frac{1-q}{\lambda}(\lambda t-x)_{+}\right)^{1 /(1-q)} \quad \text { if } 0<q<1 .
$$

It turns out that this traveling wave generates for $\varepsilon>0$ a one-parameter family of traveling wave solutions if and only if $q+\mu \geqslant 1$ (and $0<q<1$ ). All these solutions behave like 99 as $x \rightarrow \lambda t$ (i.e., they can be written in the form $u_{q}(x, t)(1+o(1))$ as $x \rightarrow \lambda t$ ), and as $\varepsilon \rightarrow 0$ they converge to (9). Similar results holds if $u^{q}$ is replaced by the Heaviside function $H(u)$ (with the condition $q+\mu \geqslant 1$ replaced by $\mu \geqslant 1$ ).

The second generalization of the porous medium equation (6) that we consider is the system

$$
u_{t}=\left(v u_{x}\right)_{x}, \quad v_{t}=\left(u v_{x}\right)_{x} .
$$

It is not difficult to see that for each wave speed $\lambda>0$ all traveling wave solutions are given by the one-parameter family

$$
u(x, t)=\frac{1}{C}\left(1-e^{-\lambda C(\lambda t-x)_{+}}\right), \quad v(x, t)=\frac{1}{C}\left(e^{\lambda C(\lambda t-x)_{+}}-1\right),
$$

where $C \neq 0$ is the parameter $\left(C=0\right.$ corresponds to $u=v=\lambda(\lambda t-x)_{+}$, the solution (7) of equation (6) with $\varepsilon=\mu=1$ ). Hence for given $\lambda>0$ the unique traveling wave solution for the 
scalar equation $u_{t}=\left(u u_{x}\right)_{x}$ is replaced by a one-parameter family in case of the system. All these traveling wave solutions behave in first approximation like $\max \{\lambda(\lambda t-x), 0\}$ near the interface $x=\lambda t$, so it could still be true that in case of general solutions the speed of the interface determines the behavior of general solutions near the moving interface.

As a conclusion we could say that in case of the porous medium equation (6) both source terms and generalizations to systems may generate one-parameter families of traveling wave solutions, at least for certain parameter values.

\section{Main results}

We look for traveling wave of solutions of (1) with positive speed $\lambda$ :

$$
(\omega(\xi), p(\xi)), \quad \xi:=x-\lambda t, \quad \lambda>0 .
$$

We suppose that $x=\lambda t$ is the interface, $\xi=0$, and in view of the general properties (2), (3) and (4) we assume that

$$
\omega(\xi)=p(\xi)=0 \quad \text { if } \xi>0
$$

and

$$
0<\omega(\xi)<1 \quad \text { and } \quad p(\xi)>I \quad \text { if } \xi_{0}<\xi<0 .
$$

Here $\xi_{0}$ is a negative constant (since we are interested in the local behavior of solutions near the interface, we focus our attention on traveling waves defined for $\xi>\xi_{0}$ ). The upper bound $\omega(\xi)<1$ is motivated by the physical interpretation of the problem. It is easy to guess from the equation of system (1) which are the natural free boundary conditions:

$$
\begin{cases}-\lambda \omega=\varepsilon^{2} \omega^{\mu}(p-I)^{\beta} \frac{\mathrm{d} \omega}{\mathrm{d} \xi} & \text { at } \xi=0, \\ -\lambda p=\omega^{\alpha} \frac{\mathrm{d} p}{\mathrm{~d} \xi} & \text { at } \xi=0 .\end{cases}
$$

This leads to the following problem.

Problem TW Let $\lambda>0$ be given. Find functions $\omega(\xi)$ and $p(\xi)$, defined and smooth in the interval $\left[\xi_{0}, 0\right)$ for some negative constant $\xi_{0}$, which satisfy

$$
\begin{gathered}
-\lambda \frac{\mathrm{d} \omega}{\mathrm{d} \xi}=\varepsilon^{2} \frac{\mathrm{d}}{\mathrm{d} \xi}\left(\omega^{\mu}(p-I)^{\beta} \frac{\mathrm{d} \omega}{\mathrm{d} \xi}\right)+a(1-\omega)(p-I)^{\gamma} \quad \text { if } \xi_{0} \leqslant \xi<0, \\
-\lambda p=\omega^{\alpha} \frac{\mathrm{d} p}{\mathrm{~d} \xi} \quad \text { if } \xi_{0} \leqslant \xi<0, \\
0<\omega<1 \quad \text { and } p>I \quad \text { if } \xi_{0} \leqslant \xi<0, \\
\frac{\mathrm{d} \omega}{\mathrm{d} \xi}<0 \quad \text { if } \xi_{0} \leqslant \xi<0, \\
\omega(p-I) \rightarrow 0 \quad \text { as } \xi \nearrow 0, \\
\varepsilon^{2} \omega^{\mu}(p-I)^{\beta} \frac{\mathrm{d} \omega}{\mathrm{d} \xi}+\lambda \omega \rightarrow 0 \quad \text { as } \xi \nearrow 0 .
\end{gathered}
$$


It is easy to prove that any solution of Problem TW is a (weak) solution of system (1) in the sense of [6] (in a neighborhood of $\xi=0$ ).

We briefly comment on the properties $[12)-(17)$ :

- (12), (13) and (17) express the equations for $\omega$ and $p$ and the interface conditions at $\xi=0$;

- (14) and (16) are motivated by the general properties (2), (3) and (10) (see also (11));

- to motivate (15), we distinguish two cases;

$$
\lim _{\xi \supset 0} \omega(\xi)=0 \quad \text { and } \quad \lim _{\xi \nearrow 0} \omega(\xi)>0
$$

(it follows from elementary BV-estimates that the limit exists and is finite). In the former case assumption (15) is natural, since $\omega>0$ if $\xi<0$. In the latter case it is enough to observe that

$$
\varepsilon^{2} \omega^{\mu}(p-I)^{\beta} \frac{\mathrm{d} \omega}{\mathrm{d} \xi}=-\lambda \omega-a \int_{0}^{\xi}(1-\omega)(p-I)^{\gamma}<0
$$

if $\xi$ is small enough.

Now we are ready to list the main results of this paper. In view of $(13)$ and $(15)$ we may define

$$
\omega^{*}:=\lim _{\xi \nearrow 0} \omega(\xi) \quad \text { and } \quad p^{*}:=\lim _{\xi \nearrow 0} p(\xi) .
$$

By 160 we can distinguish three cases: $0<\omega^{*}<1$ and $p^{*}=I, \omega^{*}=0$ and $p^{*}>I, \omega^{*}=0$ and $p^{*}=I$. The first two cases concern traveling waves with discontinuities at $\xi=0$ (for, respectively, $\omega$ and $\left.(p-I)_{+}\right)$, while the latter case treats continuous traveling waves.

THEOREM 3.1 (Traveling waves with jumps in $\omega$ ) Suppose that

$$
0<\omega^{*}<1, \quad \omega^{*}:=\lim _{\xi \nearrow 0} \omega(\xi)
$$

(i) Problem TW has a solution for some $\xi_{0}<0$ if and only if $I>0$ and $0<\beta<1$.

(ii) If $I>0$ and $0<\beta<1$ the solution of Problem TW is uniquely determined by $\omega^{*}$ and the wave speed $\lambda$, and it behaves near $\xi=0$ (for $\xi<0$ ) as follows:

$$
\omega \approx \omega^{*}+\frac{\varepsilon^{2} \lambda^{1-\beta}}{(1-\beta) I^{\beta}}\left(\omega^{*}\right)^{1+\alpha \beta-\mu}|\xi|^{1-\beta}, \quad p \approx I+\lambda\left(\omega^{*}\right)^{-\alpha} I|\xi| .
$$

THEOREM 3.2 (Traveling waves with jumps in $\left.(p-I)_{+}\right)$Suppose that

$$
p^{*}:=\lim _{\xi \nearrow 0} p(\xi)>I .
$$

(i) If $\alpha \geqslant \mu$, Problem TW has no solution.

(ii) If $\mu<1$, Problem TW has no solution.

(iii) If $\mu=1$ and $\alpha<1$, for any $\lambda>0$ and $p^{*}>I$ which satisfy

$$
4 a \varepsilon^{2}\left(p^{*}-I\right)^{\beta+\gamma}<\lambda^{2},
$$

there exist 
(a) a solution of Problem TW which behaves near $\xi=0$ (for $\xi<0$ ) as follows:

$$
\omega \approx A_{+}|\xi|, \quad p \approx p^{*}+C_{+}|\xi|^{1-\alpha}
$$

where

$$
A_{+}=\frac{\lambda}{2 \varepsilon^{2}}\left(1+\sqrt{1-\frac{4 a \varepsilon^{2}\left(p^{*}-I\right)^{\beta+\gamma}}{\lambda^{2}}}\right)\left(p^{*}-I\right)^{-\beta}, \quad C_{+}=\lambda(1-\alpha)^{-1} p^{*} A_{+}^{-\alpha} ;
$$

(b) a one-parameter family of solutions of Problem TW which behave near $\xi=0$ (for $\xi<0$ ) as follows:

$$
\omega \approx A_{-}|\xi|, \quad p \approx p^{*}+C_{-}|\xi|^{1-\alpha}
$$

where

$$
A_{-}=\frac{\lambda}{2 \varepsilon^{2}}\left(1-\sqrt{1-\frac{4 a \varepsilon^{2}\left(p^{*}-I\right)^{\beta+\gamma}}{\lambda^{2}}}\right)\left(p^{*}-I\right)^{-\beta}
$$

and $C_{-}$is defined as $C_{+}$with $A_{+}$replaced by $A_{-}$.

(iv) (a) If $\mu>1$ and $\alpha<\mu$, for any $\lambda>0$ and $p^{*}>I$ there exists a solution of Problem TW which behaves near $\xi=0$ (for $\xi<0$ ) as follows:

$$
\omega \approx A|\xi|^{1 / \mu}, \quad p \approx p^{*}+C|\xi|^{1-\alpha / \mu}
$$

where

$$
\begin{aligned}
& A=(\lambda \mu)^{1 / \mu}\left(p^{*}-I\right)^{-\beta / \mu} \varepsilon^{-2 / \mu}, \\
& C=\lambda^{(\mu-\alpha) / \mu}\left(p^{*}-I\right)^{\alpha \beta / \mu} p^{*}(\mu-\alpha)^{-1} \mu^{(\mu-\alpha) / \mu} \varepsilon^{2 \alpha / \mu} .
\end{aligned}
$$

(b) If $\mu>1$ and $\alpha<1$, for any $\lambda>0$ and $p^{*}>I$ there exists a one-parameter family of solutions of Problem TW which behave near $\xi=0$ (for $\xi<0$ ) as follows:

$$
\omega \approx A_{0}|\xi|, \quad p \approx p^{*}+C_{0}|\xi|^{1-\alpha}
$$

where

$$
A_{0}=\frac{a\left(p^{*}-I\right)^{\gamma}}{\lambda}, \quad C_{0}=\frac{\lambda^{\alpha+1} p^{*}}{a^{\alpha}\left(p^{*}-I\right)^{\alpha \gamma}(1-\alpha)} .
$$

REMARK 3.1 Observe that $A_{-} \rightarrow A_{0}$ and $C_{-} \rightarrow C_{0}$ as $\varepsilon \rightarrow 0$. As a matter of fact, it is not difficult to show that if $\varepsilon=0$, for any $\lambda>0$ and $p^{*}>I$ Problem TW has a unique solution $(\omega, p)$ which behaves, as $\xi \rightarrow 0^{-}$, as follows:

$$
\omega \approx A_{0}|\xi|, \quad p \approx p^{*}+C_{0}|\xi|^{1-\alpha}
$$

THEOREM 3.3 (Continuous traveling waves)

(i) Let $I>0$. If

$$
\beta>1 \quad \text { and } \alpha>\max \left\{\mu, \frac{\mu(\gamma+1)+(\beta-1)}{\beta+\gamma}\right\}
$$


then Problem TW has a one-parameter family of solutions which behave near $\xi=0$ (for $\xi<0)$ as follows:

$$
\omega \approx A|\xi|^{\frac{\beta-1}{\alpha \beta-\mu}}, \quad p \approx I+C|\xi|^{\frac{\alpha-\mu}{\alpha \beta-\mu}}
$$

where

$$
\begin{aligned}
& A=\left(\varepsilon^{-4 \beta-2} \lambda^{\beta-1} I^{\beta}|\alpha \beta-\mu|^{\beta-1}|\beta-1||\alpha-\mu|^{-\beta}\right)^{\frac{1}{\alpha \beta-\mu}} \\
& C=\left(\varepsilon^{\frac{-2 \alpha \beta-2 \alpha+4 \mu \beta-2 \mu}{\beta-1}} \lambda^{\alpha-\mu} I^{-\mu}|\alpha \beta-\mu|^{-\mu+\alpha}|\beta-1|^{-\alpha}|\alpha-\mu|^{\mu}\right)^{\frac{1}{\alpha \beta-\mu}} .
\end{aligned}
$$

(ii) Let $I=0$. If

$$
\alpha>\max \left\{\mu, \frac{\mu \gamma+\beta}{\beta+\gamma}\right\}
$$

then Problem TW has a one-parameter family of solutions which behave near $\xi=0(\xi<0)$ as

$$
\omega \approx A|\xi|^{1 / \alpha}, \quad p \approx C|\xi|^{\frac{\alpha-\mu}{\alpha \beta}}
$$

where

$$
\begin{aligned}
& A=\left(\varepsilon^{\frac{2(1-\beta)}{\beta+\gamma}} \lambda \alpha \beta(\alpha-\mu)^{-1}\right)^{1 / \alpha}, \\
& C=\left(\varepsilon^{\frac{2(\beta \mu-\beta \alpha-\mu+\alpha \gamma)}{\beta+\gamma}} \lambda^{\alpha-\mu} \beta^{-\mu} \alpha^{\alpha-\mu}(\alpha-\mu)^{\mu}\right)^{1 / \alpha \beta} .
\end{aligned}
$$

(iii) Let $I>0$. If

$$
\alpha<\min \left\{1, \frac{\mu(\gamma+1)+\beta-1}{\beta+\gamma}\right\}
$$

then Problem TW has a one-parameter family of solutions which behave near $\xi=0$ (for $\xi<0)$ as follows:

$$
\omega \approx A|\xi|^{\frac{\gamma+1}{\alpha \gamma+1}}, \quad p \approx I+C|\xi|^{\frac{1-\alpha}{\alpha \gamma+1}}
$$

where

$$
\begin{aligned}
& A=C^{\gamma} \frac{a}{\lambda} \frac{\alpha \gamma+1}{\gamma+1}, \\
& C=\left(\lambda^{1+\alpha} I^{-\alpha}(\alpha \gamma+1)^{1-\alpha}(\gamma+1)^{\alpha}(1-\alpha)^{-1}\right)^{\frac{1}{\alpha \gamma+1}} .
\end{aligned}
$$

(iv) Let $I=0$. If

$$
\alpha<\min \left\{1, \frac{\mu \gamma+\beta}{\beta+\gamma}\right\}
$$

then Problem TW has a one-parameter family of solutions which behave near $\xi=0$ (for $\xi<0$ ) as follows:

$$
\omega \approx A|\xi|^{1 / \alpha} \quad p \approx C|\xi|^{\frac{1-\alpha}{\alpha \gamma}}
$$

where

$$
A=\left(\frac{\lambda \gamma \alpha}{1-\alpha}\right)^{1 / \alpha}, \quad C=\left(\alpha^{1-\alpha} \gamma \lambda^{1+\alpha}(1-\alpha)^{-1} a^{-\alpha}\right)^{1 / \gamma \alpha}
$$


REMARK 3.2 Observe that in (iii) and (iv) the behavior of the traveling waves does not depend on $\varepsilon$. It is not difficult to show that if $\varepsilon=0$, for any $\lambda>0, \alpha<1$ and $I \geqslant 0$ Problem TW has a unique solution $(\omega, p)$ which behaves, as $\xi \rightarrow 0^{-}$, like the solutions of Theorem 3.3 (iii) (if $I>0$ ) and Theorem 3.3 (iv) (if $I=0$ ).

REMARK 3.3 Conditions (19), (21), (22) and (23) can be reformulated, respectively, as

$$
\begin{aligned}
& \beta>1 \text { and } \quad \begin{cases}\alpha>\mu & \text { if } \mu \geqslant 1, \\
(\gamma+1) \mu+\beta<\alpha(\beta+\gamma)+1 & \text { if } \mu<1,\end{cases} \\
& \begin{cases}\alpha>\mu & \text { if } \mu \geqslant 1, \\
\alpha(\beta+\gamma)>\mu \gamma+\beta & \text { if } \mu<1,\end{cases} \\
& \begin{cases}\alpha<1 & \text { if } \mu \geqslant 1, \\
\alpha(\beta+\gamma)<\mu(\gamma+1) & \text { if } \mu<1, \\
\alpha(\beta+\gamma)<\mu \gamma+\beta & \text { if } \mu \geqslant 1,\end{cases} \begin{cases}\alpha<1 & \text { if } \mu<1,\end{cases}
\end{aligned}
$$

In view of the discussion in Section 2 and Remarks 3.1 and 3.2 , it is not difficult to give an interpretation of the strong parameter dependence of the number of traveling wave solutions and their behavior near the interface. The one-parameter families of solutions defined by Theorem 3.1 (ii) ( $\omega^{*}$ is the parameter), Theorem 3.2(iii)(a) and (iv)(a) ( $p^{*}$ is the parameter) and Theorem 3.3 (i) and (ii) are generated, for certain parameter values, by the fact that we deal with a system of diffusion equations (observe that the coefficients which determine the local behavior strongly depend on $\varepsilon$, but hardly on $a$ ). It follows from Remark 3.2 that the one-parameter families defined by Theorem 3.3(iii) and (iv) are generated by the source term in the equation for $\omega$ and the unique solution if $\varepsilon=0$ (see Section 2 for a comparison with the solutions generated by $u_{q}$ ). Similarly, concerning the solutions defined by Theorem 3.2(iii)(b), and (iv)(b) it follows from Remark 3.1 that for $\varepsilon=0$ a one-parameter family of solutions is generated by a system without diffusion for $\omega$ ( $p^{*}$ is the parameter), while for $\varepsilon>0$ this one-parameter family becomes a two-parameter family.

\section{A change of variables}

In view of (15), we can introduce $\log \omega$ as an independent variable:

$$
y=\log \omega .
$$

As new dependent variables we use

$$
\begin{aligned}
& u=-\frac{\varepsilon^{2}}{\lambda} \omega^{\mu-1}(p-I)^{\beta} \frac{\mathrm{d} \omega}{\mathrm{d} \xi}>0, \\
& v=\int \frac{1}{p(p-I)^{\beta}} \mathrm{d} p .
\end{aligned}
$$

We observe that

$$
\int_{I} \frac{1}{p(p-I)^{\beta}} \mathrm{d} p>-\infty \Leftrightarrow I>0 \text { and } 0<\beta<1
$$

and in this case we specify the primitive in 26 :

$$
v=\int_{I}^{p} \frac{1}{s(s-I)^{\beta}} \mathrm{d} s \quad \text { if } I>0 \text { and } 0<\beta<1 .
$$


We observe that

$$
\text { 17) } \Leftrightarrow \omega(u-1)=0 \text { at } \xi=0 \text {, }
$$

which implies that

$$
\begin{aligned}
u=1 \quad \text { at } y=\log (\omega(0)) & \text { if } \omega>0 \text { at } \xi=0, \\
e^{y} u(y) \rightarrow 0 & \text { as } y \rightarrow-\infty \text { if } \omega=0 \text { at } \xi=0 .
\end{aligned}
$$

To determine the equation for $u(y)$ we observe that

$$
\frac{\mathrm{d} u}{\mathrm{~d} y}=\frac{\mathrm{d} u}{\mathrm{~d} \omega} \frac{\mathrm{d} \omega}{\mathrm{d} y}=\omega \frac{\mathrm{d} u}{\mathrm{~d} \omega}=-u+\frac{\mathrm{d}(\omega u)}{\mathrm{d} \omega}=-u-\frac{\varepsilon^{2}}{\lambda} \frac{\mathrm{d}}{\mathrm{d} \xi}\left(\omega^{\mu}(p-I)^{\beta} \frac{\mathrm{d} \omega}{\mathrm{d} \xi}\right) \frac{1}{\frac{\mathrm{d} \omega}{\mathrm{d} \xi}}
$$

Using relations (12), 24) and (25) we obtain

$$
\begin{aligned}
\frac{\mathrm{d} u}{\mathrm{~d} y} & =-u-\frac{1}{\lambda}\left(-\lambda \frac{\mathrm{d} \omega}{\mathrm{d} \xi}-a(1-\omega)(p-I)^{\gamma}\right) \frac{1}{\frac{\mathrm{d} \omega}{\mathrm{d} \xi}} \\
& =1-u-\frac{a}{\lambda^{2}}(1-\omega) \omega^{\mu-1}(p-I)^{\beta+\gamma} \frac{\varepsilon^{2}}{u} \\
& =1-u-\frac{\varepsilon^{2} a}{\lambda^{2}}\left(1-e^{y}\right) e^{(\mu-1) y} \frac{h(v)}{u}
\end{aligned}
$$

where we have set

$$
h(v):=(p-I)^{\beta+\gamma} .
$$

The equation for $v(y)$ follows at once from (13, , 24, 25) and 26):

$$
\frac{\mathrm{d} v}{\mathrm{~d} y}=\frac{\mathrm{d} v}{\mathrm{~d} p} \cdot \frac{\mathrm{d} p}{\mathrm{~d} \xi} \cdot \frac{\mathrm{d} \xi}{\mathrm{d} \omega} \cdot \frac{\mathrm{d} \omega}{\mathrm{d} y}=\frac{1}{p(p-I)^{\beta}} \cdot \frac{-\lambda p}{\omega^{\alpha}} \cdot \frac{\varepsilon^{2} \omega^{\mu-1}(p-I)^{\beta}}{-\lambda u} \cdot \omega=\frac{\varepsilon^{2} \omega^{\mu-\alpha}}{u}=\frac{\varepsilon^{2}}{u} e^{(\mu-\alpha) y} .
$$

The proof of the main result is based on the analysis of the system for $u(y)$ and $v(y)$ :

$$
\left\{\begin{array}{l}
\frac{\mathrm{d} u}{\mathrm{~d} y}=1-u-\frac{\varepsilon^{2} a}{\lambda^{2}}\left(1-e^{y}\right) e^{(\mu-1) y} \frac{1}{u} h(v) \\
\frac{\mathrm{d} v}{\mathrm{~d} y}=\frac{\varepsilon^{2}}{u} e^{(\mu-\alpha) y}
\end{array}\right.
$$

\section{Traveling waves with jumps in $\omega$ : proof of Theorem 3.1}

Since $\omega^{*}>0$ it follows from 16 that

$$
p \rightarrow I \quad \text { as } \xi \rightarrow 0
$$

Setting $y^{*}=\log \omega^{*}>-\infty$, we see from (24) that we have to look for solutions of system (32) for $y^{*} \leqslant y \leqslant y_{0}$ for some $y_{0}>y^{*}$ which satisfy (see 29]) the condition

$$
u\left(y^{*}\right)=1 .
$$


It follows from (26), (27), (28) and $(33)$ that

$$
v\left(y^{*}\right)= \begin{cases}0 & \text { if } I>0 \text { and } 0<\beta<1, \\ -\infty & \text { otherwise. }\end{cases}
$$

On the other hand, by (32),

$$
\frac{\mathrm{d} v}{\mathrm{~d} y} \rightarrow \frac{\varepsilon^{2}}{u\left(y^{*}\right)} e^{(\mu-\alpha) y^{*}}=\varepsilon^{2} e^{(\mu-\alpha) y^{*}} \quad \text { as } y \rightarrow y^{*},
$$

and hence there exists no solution such that $v\left(y^{*}\right)=-\infty$. It remains to consider the case $I>0$ and $0<\beta<1$, and it follows at once that there exists a unique solution of (32) satisfying $u\left(y^{*}\right)=1$ and $v\left(y^{*}\right)=1$. It is straightforward to check the correspondence between the solutions $(u(y), v(y))$ and $(\omega(\xi), p(\xi))$. The local behavior of $\omega$ and $p$ follows easily from the relations

$$
\omega-\omega^{*} \approx \frac{\varepsilon^{2}\left(\omega^{*}\right)^{1+\alpha-\mu}}{(1-\beta) I}(p-I)^{1-\beta} \text { and } \frac{\mathrm{d} p}{\mathrm{~d} \xi} \approx-\lambda\left(\omega^{*}\right)^{-\alpha} p .
$$

The latter relation follows from 13 and the former from

$$
\begin{array}{ll}
v \approx \frac{1}{(1-\beta) I}(p-I)^{1-\beta} & (\text { by [28) }), \\
v \approx \varepsilon^{2}\left(\omega^{*}\right)^{\mu-\alpha}\left(y-y^{*}\right) & \text { (by (34) }),
\end{array}
$$

and

$$
y-y^{*}=\log \frac{\omega}{\omega^{*}} \approx \frac{\omega-\omega^{*}}{\omega^{*}} .
$$

6. Traveling waves with a jump in $(p-I)_{+}$: proof of Theorem 3.2

Since $p^{*}>I,(16)$ implies that $\omega \rightarrow 0$ as $\xi \nearrow 0$. Let $v^{*}$ be the value of $v$ (see $\left.\sqrt{26}-\sqrt{28}\right)$ ) which corresponds to $p^{*}$. We look for solutions of system $\left.\sqrt{32}\right)$ in $\left(-\infty, y_{0}\right)$ for some $-\infty<y_{0}<0$ such that

$$
v \rightarrow v^{*} \quad \text { as } y \rightarrow-\infty .
$$

We claim that

$$
u(y)<1 \quad \text { for all } y<y_{0} .
$$

Indeed, if $u\left(y_{1}\right) \geqslant 1$ for some $y_{1}<y_{0}$, then (32) implies that

$$
\frac{\mathrm{d} u}{\mathrm{~d} y} \leqslant 1-u \quad \text { in }\left(-\infty, y_{1}\right)
$$

and hence $(u-1) e^{y}$ is nonincreasing in $\left(-\infty, y_{1}\right)$. Since $(u-1) e^{y} \geqslant 0$ at $y=y_{1}$ and $u \neq 1$ in $\left(-\infty, y_{1}\right)$, this means that $e^{y} u(y)$ does not vanish as $y \rightarrow-\infty$. This contradicts 30).

Proof of (ii). It follows from (32) and (36) that

$$
\frac{\mathrm{d} v}{\mathrm{~d} y} \geqslant \varepsilon^{2} e^{(\mu-\alpha) y} .
$$

If $\alpha \geqslant \mu$ we conclude that $v \rightarrow-\infty$ as $y \rightarrow-\infty$, a contradiction with 35]. 
Proof of (ii). We set

$$
X(\omega)=\omega u(\log \omega) .
$$

In view of $30, X(\omega) \rightarrow 0$ as $\omega \rightarrow 0$. In addition, $X$ satisfies the equation

$$
\frac{\mathrm{d}}{\mathrm{d} \omega}\left(X^{2}\right)=2 X \frac{\mathrm{d}}{\mathrm{d} y}\left(e^{y} u\right) \frac{\mathrm{d} y}{\mathrm{~d} \omega}=2 X \frac{1}{\omega}\left(\omega u+\omega\left(1-u-\frac{\varepsilon^{2} a}{\lambda^{2}}(1-\omega) \omega^{\mu-1}(p-I)^{\beta+\gamma} \frac{1}{u}\right)\right),
$$

where we have used (24), (31) and (32). Hence

$$
\frac{\mathrm{d}}{\mathrm{d} \omega}\left(X^{2}\right)=2 X-\frac{2 \varepsilon^{2} a}{\lambda^{2}}(1-\omega) \omega^{\mu}(p-I)^{\beta+\gamma} \leqslant 2\left(\omega-C \omega^{\mu}\right)
$$

for some positive constant $C$. Here we have used the fact that $X \leqslant \omega$, by [36], and that $1-\omega \rightarrow 1$ and $p-I \rightarrow p^{*}-I>0$ as $\omega \rightarrow 0$. Hence $\frac{\mathrm{d}}{\mathrm{d} \omega}\left(X^{2}\right)<0$ for $\omega$ small enough; but this is not possible, since $X(0)=0$.

Proof of (iii). We look first for solutions $u(y)$ which tend to some constant $u^{*} \in(0,1]$ as $y \rightarrow-\infty$. Since

$$
\frac{\mathrm{d} u}{\mathrm{~d} y}=1-u-\frac{\varepsilon^{2} a}{\lambda^{2}}\left(1-e^{y}\right) \frac{h(v)}{u}
$$

and since, by (35), $v \rightarrow v^{*}$ as $y \rightarrow-\infty$, we expect that $u^{*}$ is a solution of the quadratic equation

$$
u^{2}-u+\frac{\varepsilon^{2} a h\left(v^{*}\right)}{\lambda^{2}}=0 .
$$

If $4 \varepsilon^{2} a h\left(v^{*}\right)<\lambda^{2}$, which, in view of the definition of $h(v)$, is equivalent to 18 , equation 37 has two solutions:

$$
u_{ \pm}=\frac{1}{2} \pm \frac{1}{2} \sqrt{1-\frac{4 \varepsilon^{2} a h\left(v^{*}\right)}{\lambda^{2}}}
$$

We observe that

$$
0<u_{-}<1 / 2<u_{+}<1
$$

Set

$$
z=\left(v-v^{*}\right) e^{(\alpha-1) y}, \quad v=v^{*}+z e^{(1-\alpha) y} .
$$

Then $u$ and $z$ satisfy the system

$$
\left\{\begin{array}{l}
\frac{\mathrm{d} u}{\mathrm{~d} y}=1-u-\frac{\varepsilon^{2} a}{\lambda^{2}}\left(1-e^{y}\right) \frac{1}{u} h\left(v^{*}+z e^{(1-\alpha) y}\right), \\
\frac{\mathrm{d} z}{\mathrm{~d} y}=\frac{\varepsilon^{2}}{u}-(1-\alpha) z
\end{array}\right.
$$

If $u \rightarrow u_{ \pm}$as $y \rightarrow-\infty$, asymptotically the system becomes

$$
\left\{\begin{array}{l}
\frac{\mathrm{d} u}{\mathrm{~d} y}=f(u, z):=1-u-\frac{\varepsilon^{2} a h\left(v^{*}\right)}{\lambda^{2} u}, \\
\frac{\mathrm{d} z}{\mathrm{~d} y}=g(u, z):=\frac{\varepsilon^{2}}{u}-(1-\alpha) z
\end{array}\right.
$$


System (39) has two equilibrium points:

$$
\begin{aligned}
& \left(u_{+}, z_{+}\right):=\left(\frac{1}{2}+\frac{1}{2} \sqrt{1-\frac{4 \varepsilon^{2} a h\left(v^{*}\right)}{\lambda^{2}}}, \frac{\varepsilon^{2}}{(1-\alpha) u_{+}}\right), \\
& \left(u_{-}, z_{-}\right):=\left(\frac{1}{2}-\frac{1}{2} \sqrt{1-\frac{4 \varepsilon^{2} a h\left(v^{*}\right)}{\lambda^{2}}}, \frac{\varepsilon^{2}}{(1-\alpha) u_{-}}\right) .
\end{aligned}
$$

Linearizing around these equilibria we obtain the Jacobian matrix

$$
\left(\begin{array}{ll}
f_{u}\left(u_{ \pm}, z_{ \pm}\right) & f_{z}\left(u_{ \pm}, z_{ \pm}\right) \\
g_{u}\left(u_{ \pm}, z_{ \pm}\right) & g_{z}\left(u_{ \pm}, z_{ \pm}\right)
\end{array}\right)=\left(\begin{array}{cc}
-2+1 / u_{ \pm} & 0 \\
-\varepsilon^{2} / u_{ \pm}^{2} & -(1-\alpha)
\end{array}\right)
$$

which has two eigenvalues:

$$
\lambda_{1}=-(1-\alpha)<0, \quad \lambda_{2}^{ \pm}=-2+\frac{1}{u_{ \pm}}= \begin{cases}<0 & \text { if } u=u_{+} \\ >0 & \text { if } u=u_{-}\end{cases}
$$

By standard theory, system 39 has a unique solution $(u(y), z(y))$ in a neighborhood of $-\infty$ such that

$$
(u(y), z(y)) \rightarrow\left(u_{+}, z_{+}\right) \quad \text { as } y \rightarrow-\infty
$$

and a one-parameter family of solutions such that

$$
(u(y), z(y)) \rightarrow\left(u_{-}, z_{-}\right) \quad \text { as } y \rightarrow-\infty .
$$

By [9. Chapter IV, Theorems 2.1 and 3.1], the same result holds for the original nonautonomous system (38).

Going back to the original variables we obtain Theorem 3.2 iii). We observe that $h\left(v^{*}\right)=$ $\left(p^{*}-I\right)^{\beta+\gamma}$. The behavior of the solutions near $\xi=0$ follows easily from the relations

$$
\begin{aligned}
& v \approx v^{*}+\frac{1}{u_{ \pm}} \cdot \frac{\varepsilon^{2}}{1-\alpha} e^{(1-\alpha) y}=v^{*}+\frac{1}{u_{ \pm}} \cdot \frac{\varepsilon^{2}}{1-\alpha} \omega^{1-\alpha} \\
& v-v^{*} \approx \frac{1}{p^{*}\left(p^{*}-I\right)^{\beta}}\left(p-p^{*}\right) \\
& \omega^{\alpha} d p \approx-\lambda p^{*} d \xi
\end{aligned}
$$

Proof of (iv). The proof of part (a) is similar to the one of (iii). Setting

$$
z=\left(v-v^{*}\right) e^{-(\mu-\alpha) y}
$$

we obtain the system

$$
\left\{\begin{array}{l}
\frac{\mathrm{d} u}{\mathrm{~d} y}=1-u-\frac{\varepsilon^{2} a}{\lambda^{2}}\left(1-e^{y}\right) \frac{1}{u} h\left(v^{*}+e^{(\mu-\alpha) y} z\right) e^{(\mu-1) y}, \\
\frac{\mathrm{d} z}{\mathrm{~d} y}=\frac{\varepsilon^{2}}{u}-(\mu-\alpha) z .
\end{array}\right.
$$


Asymptotically, as $y \rightarrow-\infty$, the system reduces to

$$
\left\{\begin{array}{l}
\frac{\mathrm{d} u}{\mathrm{~d} y}=1-u \\
\frac{\mathrm{d} z}{\mathrm{~d} y}=\frac{\varepsilon^{2}}{u}-(\mu-\alpha) z
\end{array}\right.
$$

and the linearized system around the equilibrium point $\left(1, \varepsilon^{2} /(\mu-\alpha)\right)$ has two negative eigenvalues, -1 and $-(\mu-\alpha)$. We omit the details of the proof.

To prove part (b) we look for solutions $(u(y), v(y))$ such that

$$
u(y) \rightarrow 0 \quad \text { as } y \rightarrow-\infty .
$$

The equation for $u$ suggests that such a solution should satisfy

$$
u e^{-(\mu-1) y} \rightarrow \frac{\varepsilon^{2} a h\left(v^{*}\right)}{\lambda^{2}} .
$$

Therefore we set

$$
z=u e^{-(\mu-1) y} \quad \text { and } \quad q=\left(v-v^{*}\right) e^{-(1-\alpha) y} .
$$

This leads to the system

$$
\left\{\begin{array}{l}
\frac{\mathrm{d} z}{\mathrm{~d} y}=-\mu z+e^{-(\mu-1) y}\left(1-\frac{\varepsilon^{2} a}{\lambda^{2}}\left(1-e^{y}\right) \frac{1}{z} h\left(v^{*}+q e^{(1-\alpha) y}\right)\right), \\
\frac{\mathrm{d} q}{\mathrm{~d} y}=-(1-\alpha) q+\frac{\varepsilon^{2}}{z}
\end{array}\right.
$$

To eliminate the factor $e^{-(\mu-1) y}$ we introduce a new independent variable:

$$
t=e^{-(\mu-1) y} \rightarrow \infty \quad \text { as } y \rightarrow-\infty .
$$

Hence $z$ and $q$ satisfy

$$
\left\{\begin{array}{l}
\frac{\mathrm{d} z}{\mathrm{~d} t}=-\frac{1}{\mu-1}\left(1-\frac{\varepsilon^{2} a}{\lambda^{2}}\left(1-t^{-\frac{1}{\mu-1}}\right) \frac{1}{z} h\left(v^{*}+q t^{-\frac{1-\alpha}{\mu-1}}\right)\right)+\frac{\mu}{\mu-1} \frac{z}{t} \\
\frac{\mathrm{d} q}{\mathrm{~d} t}=\frac{1}{\mu-1}\left((1-\alpha) q-\frac{\varepsilon^{2}}{z}\right) \frac{1}{t} .
\end{array}\right.
$$

To obtain an autonomous system we introduce a third dependent variable:

$$
s^{2 n}=1 / t, \quad n \in \mathbb{N},
$$

hence

$$
s^{2 n}=1 / t \rightarrow 0 \quad \text { as } t \rightarrow \infty .
$$


This leads to the system

$$
\left\{\begin{array}{l}
\frac{\mathrm{d} z}{\mathrm{~d} t}=-\frac{1}{\mu-1}\left(1-\frac{\varepsilon^{2} a}{\lambda^{2}}\left(1-|s|^{2 n \frac{1}{\mu-1}}\right) \frac{1}{z} h\left(v^{*}+q|s|^{2 n \frac{1-\alpha}{\mu-1}}\right)\right)+\frac{\mu}{\mu-1} s^{2 n} z, \\
\frac{\mathrm{d} q}{\mathrm{~d} t}=\frac{1}{\mu-1}\left((1-\alpha) q-\frac{\varepsilon^{2}}{z}\right) s^{2 n}, \\
\frac{\mathrm{d} s}{\mathrm{~d} t}=-\frac{s^{2 n+1}}{2 n} .
\end{array}\right.
$$

The system can be written in the following way:

$$
\left\{\begin{array}{l}
\frac{\mathrm{d} \tilde{z}}{\mathrm{~d} t}=-\frac{1}{\mu-1} \frac{\lambda^{2}}{\varepsilon^{2} a h\left(v^{*}\right)} \tilde{z}+Z(\tilde{z}, \tilde{q}, s), \\
\frac{\mathrm{d} \tilde{q}}{\mathrm{~d} t}=Q(\tilde{z}, \tilde{q}, s), \\
\frac{\mathrm{d} s}{\mathrm{~d} t}=S(s) .
\end{array}\right.
$$

Here $\tilde{z}=z-\varepsilon^{2} a h\left(v^{*}\right) / \lambda^{2}, \tilde{q}=q(1-\alpha)-\lambda^{2} / a h\left(v^{*}\right)$ and $S(s) \equiv-s^{2 n+1} / 2 n$. Moreover, near the origin we have the asymptotic expansions

$$
\begin{aligned}
& Q(\tilde{z}, \tilde{q}, s) \approx \frac{(1-\alpha) s^{2 n}}{\mu-1}\left(\tilde{q}+\frac{\lambda^{4}}{\varepsilon^{2} a^{2} h^{2}\left(v^{*}\right)} \tilde{z}\right)+O\left(s^{2 n} \tilde{z}^{2}\right), \\
& Z(\tilde{z}, \tilde{q}, s) \approx O\left(|s|^{2 n \varphi}+\tilde{z}^{2}\right), \quad \varphi=\min \left\{1, \frac{1-\alpha}{\mu-1}\right\} .
\end{aligned}
$$

In system (42) we are interested in the stability of the equilibrium point $(0,0,0)$.

Let $n>(\mu-1) / 2(1-\alpha)$. Then the functions $Q, Z$ and $S$ are smooth in a neighborhood of the origin. In particular, we have

$$
Q(0,0,0)=Z(0,0,0)=S(0)=0, \quad|\nabla Q(0,0,0)|=|\nabla Z(0,0,0)|=S^{\prime}(0)=0 .
$$

Therefore we apply center manifold theory (see [7]). In view of [7, Chapter I, Theorem 1] there exists a smooth invariant manifold $\tilde{z}=L(\tilde{q}, s)$ (the so called "center manifold") such that

$$
\begin{aligned}
L(0,0) & \equiv 0, \\
|\nabla L(0,0)| & \equiv 0 .
\end{aligned}
$$

The flow on this invariant manifold is governed by the system

$$
\left\{\begin{array}{l}
\frac{\mathrm{d} \tilde{q}}{\mathrm{~d} t}=Q(L(\tilde{q}, s), \tilde{q}, s), \\
\frac{\mathrm{d} s}{\mathrm{~d} t}=S(s) .
\end{array}\right.
$$

From the asymptotic expansion of $Q$ and $(43)$ and 444 , we get

$$
\left\{\begin{array}{l}
\frac{\mathrm{d} \tilde{q}}{\mathrm{~d} t}=\frac{(1-\alpha) \tilde{q} s^{2 n}}{\mu-1}+o\left(s^{2 n+1}+|\tilde{q}| s^{2 n}\right), \\
\frac{\mathrm{d} s}{\mathrm{~d} t}=-\frac{s^{2 n+1}}{2 n}
\end{array}\right.
$$


After the change of variables $\tau=\log t=-2 n \log s$ the equations on the center manifold are

$$
\left\{\begin{array}{l}
\frac{\mathrm{d} \tilde{q}}{\mathrm{~d} \tau}=\frac{(1-\alpha) \tilde{q}}{\mu-1}+o(|s|+|\tilde{q}|) \\
\frac{\mathrm{d} s}{\mathrm{~d} \tau}=-\frac{s}{2 n}
\end{array}\right.
$$

The origin is unstable and the flux diagram of the system is represented in Figure 1. In particular, there exists a unique solution converging asymptotically to the origin as $t \rightarrow \infty$. We denote by $(\bar{z}(t), \bar{q}(t))$ the corresponding solution in the original coordinate system $(z, q)$ and in Figure 2 we represent it by $\Gamma$ in the coordinate system $(z, q, t)$.

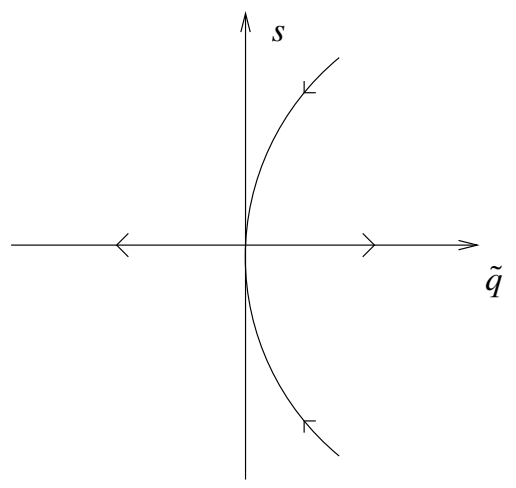

FIG. 1. The flux diagram on the center manifold.

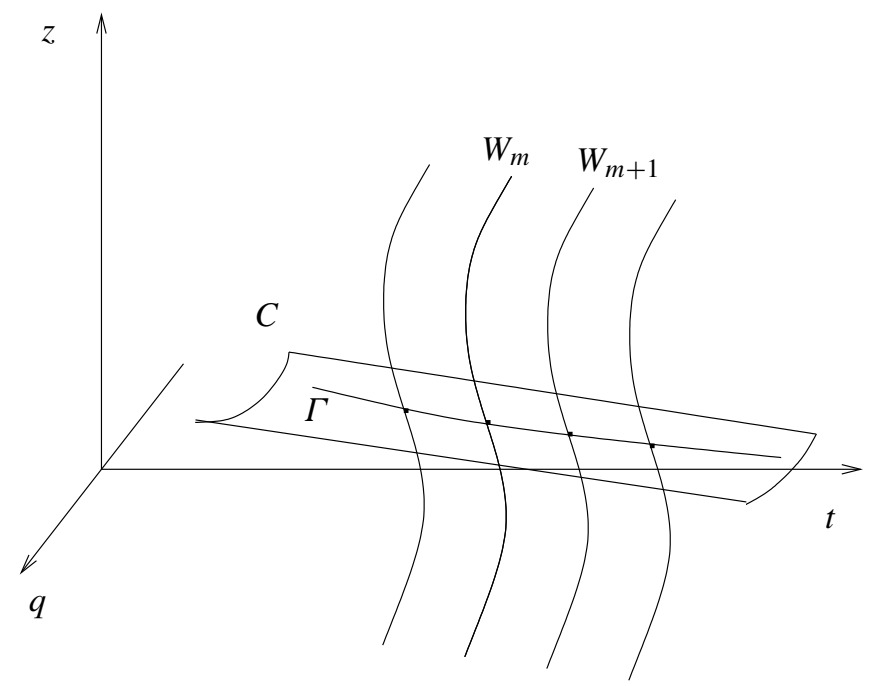

FIG. 2. The invariant manifolds $W_{m}$, and the center manifold $C$ represented in the system of coordinates $(z, q, t) ; \Gamma$ is the unique asymptotically stable trajectory on the center manifold. 
Our goal is to show that there is a one-parameter family of solutions which converge asymptotically to $\left(\varepsilon^{2} a h\left(v^{*}\right) / \lambda^{2}, \lambda^{2} / a(1-\alpha) h\left(v^{*}\right)\right)$ as $t \rightarrow \infty$. In order to achieve this result we rewrite the equations for the new variables $x_{1}=z-\bar{z}$ and $x_{2}=q-\bar{q}$. The system assumes the following general form:

$$
\dot{X}=F(X, t), \quad X=\left(\begin{array}{l}
x_{1} \\
x_{2}
\end{array}\right) .
$$

We denote by $\Phi$ the flux of our system, so that

$$
X(t)=\Phi\left(X_{0} ; t, t_{0}\right)
$$

is the trajectory at time $t$ which is equal to $X_{0}$ at time $t_{0}$. Hence we consider a family of time-discrete fluxes

$$
\Phi_{m}(X)=\Phi\left(X_{m} ; t_{m+1}, t_{m}\right)
$$

where $t_{i+1}=t_{i}+\Delta t$. We can now write the family in the form

$$
\Phi_{m}(X)=A_{m} X+\alpha_{m}(X),
$$

where $A_{m}$ denotes the exponential matrix

$$
\left.\exp \int_{t_{m}}^{t_{m+1}} \mathcal{J} F(X, t)\right|_{X=0} \mathrm{~d} t \quad(\mathcal{J} \text { is the Jacobian })
$$

and $\alpha_{m}(X)=O\left(|X|^{2}\right)$ contains the higher order terms and is $C_{\text {loc }}^{1}$-bounded uniformly in $m$. We claim that in our case for $m$ large enough the matrices $A_{m}$ have a spectral gap, i.e. there are two constants $v_{1}<1 \leqslant v_{2}$ such that the eigenvalues $\lambda_{1 m}$ and $\lambda_{2 m}$ satisfy $\lambda_{1 m} \leqslant v_{1}<v_{2} \leqslant \lambda_{2 m}$ for $m$ large enough.

Indeed, $\left.\mathcal{J} F(X, t)\right|_{X=0}$ is nothing but the Jacobian of the map defined, for fixed $t$, by the right hand side of 40$]$, evaluated at $(\bar{z}(t), \bar{q}(t))$ :

$$
\left(\begin{array}{cc}
-\frac{\varepsilon^{2} a h\left(v^{*}+\bar{q}(t) t^{-\frac{1-\alpha}{\mu-1}}\right)\left(1-t^{-\frac{1}{\mu-1}}\right)}{(\mu-1) \bar{z}(t)^{2} \lambda^{2}}+\frac{\mu t}{\mu+1} & \frac{t^{-\frac{1-\alpha}{\mu-1}} a h^{\prime}\left(v^{*}+\bar{q}(t) t^{-\frac{1-\alpha}{\mu-1}}\right)\left(1-t^{-\frac{1}{\mu-1}}\right)}{(\mu-1) \bar{z}(t) \lambda^{2}} \\
\frac{1}{t} \frac{1}{\bar{z}(t)^{2}(\mu-1)} & \frac{1-\alpha}{t(\mu-1)}
\end{array}\right) .
$$

A straightforward calculation shows that this matrix has two real eigenvalues $\delta_{1}(t)<\delta_{2}(t)$ which satisfy

$$
\delta_{1}(t) \rightarrow-\delta_{1 \infty} \equiv-\frac{\lambda^{2}}{\varepsilon^{2}(\mu-1) a h\left(v^{*}\right)}<0 \quad \text { and } \quad \delta_{2}(t)=O\left(\frac{1}{t}\right) \rightarrow 0 \quad \text { as } t \rightarrow+\infty .
$$

(Observe that $\delta_{1}(t)$ converges to the coefficient of the linear term on the right hand side of [42].) The eigenvalues of the matrix $A_{m}$ are

$$
\lambda_{1 m}=\exp \int_{t_{m}}^{t_{m+1}} \delta_{1}(t) \mathrm{d} t, \quad \lambda_{2 m}=\exp \int_{t_{m}}^{t_{m+1}} \delta_{2}(t) \mathrm{d} t
$$

and the spectral gap follows since $\lambda_{1 m} \rightarrow \exp \left(-\delta_{1 \infty} \Delta t\right)$ and $\lambda_{2 m} \searrow 1$. In these hypotheses the Perron-Hadamard theorem [10] claims that for each $m$ there exists a unique one-dimensional $C^{1}$ manifold $W_{m}$ such that: 
A. In the system of coordinates $\hat{x}_{1 m}, \hat{x}_{2 m}$ such that $A_{m} \hat{x}_{i m}=\lambda_{i m} \hat{x}_{i m}$, the manifold can be represented as a graph:

$$
W_{m} \equiv\left\{\left(\hat{x}_{1 m}, \phi_{m}\left(\hat{x}_{1 m}\right)\right)\right\} ;
$$

B. $\Phi_{m}\left(W_{m}\right)=W_{m+1}$;

C. $W_{m}=\left\{X \in \mathbb{R}^{2}:\left\|\Phi_{m+L} \circ \cdots \circ \Phi_{m}(X)\right\| \stackrel{L \rightarrow \infty}{\longrightarrow} 0\right\}$;

D. The family of manifolds depends continuously in the $C^{1}$ topology on the family $\Phi_{m}$.

E. The tangent set of $\phi_{m}$ is invariant under the action of the differential of $\Phi_{m}$.

It follows from $\mathrm{D}$ that in the three-dimensional space $(z, q, t)$ (see Figure 2) we obtain a twodimensional $C^{1}$ manifold $W$ given by the union of all the $W_{m}$ obtained by varying continuously the time discretization $\Delta t$. In Figure 2 we denote the center manifold by $C$. The manifold $W$ is transversal to the center manifold and the intersection is $\Gamma$. All the trajectories contained in $W$ converge asymptotically to $\Gamma$ (and hence to $\left.\left(\varepsilon^{2} a h\left(v^{*}\right) / \lambda^{2}, \lambda^{2} / a(1-\alpha) h\left(v^{*}\right), \infty\right)\right)$ and form a oneparameter family. Our proof is complete if we can prove that these are all the trajectories satisfying this property.

By contradiction we denote by $\bar{\Gamma}$ such a trajectory which does not belong to $W$. Applying again the Perron-Hadamard theorem we obtain another $C^{1}$ two-dimensional manifold $\bar{W}$ containing $\bar{\Gamma}$. Since the eigenvectors corresponding to $\delta_{1}(t)$ (or $\lambda_{1 m}$ ) asymptotically, as $t \rightarrow \infty$ (or as $m \rightarrow+\infty$ ), point into the $z$-direction, it follows from $\mathrm{E}$ that, as $t \rightarrow \infty$, the $z$-direction is tangent to the invariant manifold $W$ at the equilibrium point. Moreover, by (44), the $z$-direction is orthogonal to the center manifold. The intersection of $C$ and $\bar{W}$ is a solution of the problem, which converges asymptotically to the equilibrium. By the uniqueness of the asymptotically stable solution on the center manifold, $\bar{W}$ intersects $C$ in $\Gamma$. Hence there are two distinct two-dimensional invariant manifolds $W$ and $\bar{W}$ given by the Perron-Hadamard theorem and intersecting on $\Gamma$, and we have a contradiction.

The asymptotic behavior of the solutions as $\xi \nearrow 0$ follows easily from the relations

$$
\begin{gathered}
v \approx v^{*}+\frac{\lambda^{2}}{a\left(p^{*}-I\right)^{\beta+\gamma}(1-\alpha)} \omega^{1-\alpha} \quad\left(\text { since } \frac{\mathrm{d} v}{\mathrm{~d} y}=\frac{\varepsilon^{2}}{z} e^{(1-\alpha) y} \approx \frac{\lambda^{2}}{a\left(p^{*}-I\right)^{\beta+\gamma}} e^{(1-\alpha) y}\right), \\
v-v^{*} \approx \frac{1}{p^{*}\left(p^{*}-I\right)^{\beta}}\left(p-p^{*}\right),
\end{gathered}
$$

and $\omega^{\alpha} d p \approx-\lambda p^{*} d \xi$.

\section{Continuous traveling waves: proof of Theorem 3.3}

Proof of (i). Let $h(v)=(p-I)^{\gamma+\beta}$ (cf. 31) $)$. Set

$$
H(y)=h(v(y)) e^{-k y}
$$

for some $k>0$, and consider $u$ and $H$ satisfying

$$
\left\{\begin{array}{l}
\frac{\mathrm{d} u}{\mathrm{~d} y}=1-u-\frac{\varepsilon^{2} a}{\lambda^{2}}\left(1-e^{y}\right) \frac{1}{u} H e^{(k+\mu-1) y}, \\
\frac{\mathrm{d} H}{\mathrm{~d} y}=-k H+p(\beta+\gamma) \frac{\varepsilon^{2}}{u} H^{\frac{2 \beta+\gamma-1}{\beta+\gamma}} e^{\left(\mu-\alpha+k \frac{\beta-1}{\beta+\gamma}\right) y} .
\end{array}\right.
$$


Choosing

$$
k=\frac{(\alpha-\mu)(\beta+\gamma)}{\beta-1}
$$

reduces the latter equation to

$$
\frac{\mathrm{d} H}{\mathrm{~d} y}=-k H+p(\beta+\gamma) \frac{\varepsilon^{2}}{u} H^{\frac{2 \beta+\gamma-1}{\beta+\gamma}} .
$$

If condition (19) is satisfied, then

$$
k>0 \text { and } k+\mu-1>0 .
$$

It is natural to look for solutions $u(y) \rightarrow 1$ as $y \rightarrow-\infty$. In this case asymptotically, as $y \rightarrow-\infty$, the system reduces to

$$
\left\{\begin{array}{l}
\frac{\mathrm{d} u}{\mathrm{~d} y}=1-u, \\
\frac{\mathrm{d} H}{\mathrm{~d} y}=-k H+\varepsilon^{2} I(\beta+\gamma) H^{\frac{2 \beta+\gamma-1}{\beta+\gamma}},
\end{array}\right.
$$

which has the equilibrium point

$$
(u, H)=\left(1,\left(\varepsilon^{2} I(\beta-1)(\alpha-\mu)^{-1}\right)^{\frac{\beta+\gamma}{1-\beta}}\right) .
$$

Linearizing around the equilibrium leads to one negative and one positive eigenvalue $(\beta>1)$. If we argue as in the previous sections, the rest of the proof is straightforward.

Proof of (ii). As in the proof of (i), system 46 is replaced by

$$
\left\{\begin{array}{l}
\frac{\mathrm{d} u}{\mathrm{~d} y}=1-u-\frac{\varepsilon^{2} a}{\lambda^{2}}\left(1-e^{y}\right) \frac{1}{u} H e^{(k+\mu-1) y}, \\
\frac{\mathrm{d} H}{\mathrm{~d} y}=-k H+(\beta+\gamma) \frac{\varepsilon^{2}}{u} H^{2 \beta+\gamma / \beta+\gamma} e^{\left(\mu-\alpha+k \frac{\beta}{\beta+\gamma}\right) y},
\end{array}\right.
$$

and we choose

$$
k=\frac{(\alpha-\mu)(\beta+\gamma)}{\beta} .
$$

By (21), $k>0$ and $k+\mu-1>0$, and linearizing around the asymptotic equilibrium point

$$
\left(1,\left(\frac{\alpha-\mu}{\varepsilon^{2} \beta}\right)^{1+\gamma / \beta}\right)
$$

yields one positive and one negative eigenvalue. We leave the details to the reader.

Proof of (iii). Let $u$ and $h$ be defined as before. We look for solutions such that $u(y)$ vanishes as $y \rightarrow-\infty$, and set

$$
\varphi(y)=e^{-r y} u(y), \quad \psi(y)=e^{-\rho y} h(v(y)),
$$


where $r$ and $\rho$ are positive constants to be determined. Then $\varphi$ and $\psi$ satisfy

$$
\left\{\begin{array}{l}
\frac{\mathrm{d} \varphi}{\mathrm{d} y}=-(r+1) \varphi+e^{-r y}\left(-\frac{\varepsilon^{2} a}{\lambda^{2}}\left(1-e^{y}\right) \frac{\psi}{\varphi} e^{(\mu-1+\rho-r) y}\right) \\
\frac{\mathrm{d} \psi}{\mathrm{d} y}=-\rho \psi+\left(I+\psi^{\frac{1}{\beta+\gamma}} e^{\frac{\rho}{\beta+\gamma} y}\right)(\beta+\gamma) \frac{\varepsilon^{2}}{\varphi} \psi^{\frac{2 \beta+\gamma-1}{\beta+\gamma}} e^{\left(\mu-\alpha+\rho \frac{\beta-1}{\beta+\gamma}-r\right) y} .
\end{array}\right.
$$

Choosing $r$ and $\rho$ such that

$$
\mu-1+\rho-r=\mu-\alpha+\rho \frac{\beta-1}{\beta+\gamma}-r=0,
$$

we obtain

$$
\rho=\frac{(\beta+\gamma)(1-\alpha)}{\gamma+1} \quad \text { and } \quad r=\mu-\alpha+\frac{(1-\alpha)(\beta-1)}{\gamma+1} .
$$

We observe that $\rho>0$ (since $\alpha<1)$ and $r>0$ (since, by [22), $(\gamma+1) r=\mu(\gamma+1)+\beta-1-$ $\alpha(\beta+\gamma)>0)$.

We introduce the new independent variable

$$
t=e^{-r y} \rightarrow+\infty \quad \text { as } y \rightarrow-\infty .
$$

Then

$$
\left\{\begin{array}{l}
\frac{\mathrm{d} \varphi}{\mathrm{d} t}=\Phi(\varphi, \psi, t):=\frac{r+1}{r t} \varphi-\frac{1}{r}\left(1-\frac{\varepsilon^{2} a}{\lambda^{2}}\left(1-t^{-1 / r}\right) \frac{\psi}{\varphi}\right), \\
\frac{\mathrm{d} \psi}{\mathrm{d} t}=\frac{1}{t} \Psi(\varphi, \psi, t):=\frac{\psi}{r t}\left(\rho-\left(I+\psi^{\frac{1}{\beta+\gamma}} t^{-\frac{\rho}{r(\beta+\gamma)}}\right)(\beta+\gamma) \frac{\varepsilon^{2}}{\varphi} \psi^{\frac{\beta-1}{\beta+\gamma}}\right) .
\end{array}\right.
$$

Since $\Phi\left(\varphi^{*}, \psi^{*},+\infty\right)=\Psi\left(\varphi^{*}, \psi^{*},+\infty\right)=0$ if we set

$$
\varphi^{*}=\frac{\varepsilon^{2} a}{\lambda^{2}} \psi^{*} \quad \text { and } \quad \psi^{*}=\left(\frac{\lambda^{2} I(\gamma+1)}{a(1-\alpha)}\right)^{\frac{\beta+\gamma}{\gamma+1}},
$$

it is natural to look for solutions such that

$$
\varphi(r) \rightarrow \varphi^{*} \quad \text { and } \quad \psi(t) \rightarrow \psi^{*} \quad \text { as } t \rightarrow \infty .
$$

The structure of the system (47) is very similar to the one of 407 and we proceed as in the proof of Theorem 3.2 iv (b). Linearizing around $\left(\varphi^{*}, \psi^{*}\right)$, we obtain a matrix which, for large $t$, behaves like

$$
\left(\begin{array}{cc}
-\frac{1}{r \varphi^{*}} & \frac{\varepsilon^{2} a}{\lambda^{2} r \varphi^{*}} \\
\frac{\lambda^{2}(1-\alpha)(\beta+\gamma)}{\varepsilon^{2} a(\gamma+1) r t} & \frac{(1-\beta)(1-\alpha)}{(\gamma+1) r t}
\end{array}\right)
$$

Introducing $s=t^{-1 / n}$ for $n$ large enough, one easily proves the existence of a smooth twodimensional center manifold which, at $\left(\varphi^{*}, \psi^{*}, 0\right)$, is orthogonal to the vector $\left(-\lambda^{2}, \varepsilon^{2} a, 0\right)$. On 
replacing $t$ by $\tau=\log t$, the linear approximation of the equation for $\psi(\tau)-\psi^{*}$ and $s(\tau)$ on the center manifold is governed by the matrix

$$
\left(\begin{array}{cc}
\frac{1-\alpha}{r} & 0 \\
0 & -\frac{1}{n}
\end{array}\right)
$$

Since $1-\alpha>0$, the center manifold contains a unique solution converging to $\left(\varphi^{*}, \psi^{*}\right)$. This defines a solution $(\bar{\varphi}, \bar{\psi})$ converging to $\left(\varphi^{*}, \psi^{*}\right)$ as $t \rightarrow \infty$. The Jacobian matrix of the map defined, for fixed $t$, by the right hand side of 47 , evaluated at $(\bar{\varphi}(t), \bar{\psi}(t))$, is given by the matrix

$$
\left(\begin{array}{cc}
\frac{r+1}{r t}-\frac{\varepsilon^{2} a\left(1-t^{-1 / r}\right) \bar{\psi}}{r \lambda^{2} \bar{\varphi}^{2}} & \frac{\varepsilon^{2} a\left(1-t^{-1 / r}\right)}{r \lambda^{2} \bar{\varphi}} \\
\frac{\varepsilon^{2}(\beta+\gamma)\left(I+\bar{\psi} \frac{1}{\beta+\gamma} t-\frac{\rho}{r(\beta+\gamma)}\right) \bar{\psi}^{1+\frac{\beta-1}{\beta+\gamma}}}{r t \bar{\varphi}^{2}} & \frac{\rho}{r t}-\frac{\varepsilon^{2}(2 \beta+\gamma-1)\left(I+\bar{\psi} \frac{1}{\beta+\gamma}-\frac{\rho}{r(\beta+\gamma)}\right) \bar{\psi} \frac{\beta-1}{\beta+\gamma}}{r t \bar{\varphi}}+\frac{\varepsilon^{2} \bar{\psi} \frac{\beta}{\beta+\gamma}}{r t \frac{\rho}{r(\gamma+\beta)} \bar{\varphi}}
\end{array}\right) .
$$

In particular, as $t \rightarrow \infty$, its trace converges to $-1 / r \varphi^{*}<0$ and its determinant behaves as $-(1-\alpha) / r^{2} t \varphi^{*}<0$. Hence it has two real eigenvalues $\delta_{1}(t)<0<\delta_{2}(t)$ which satisfy

$$
\delta_{1}(t) \rightarrow-\frac{1}{r \varphi^{*}} \quad \text { and } \quad \delta_{2}(t)=O\left(\frac{1}{t}\right) \rightarrow 0 \quad \text { as } t \rightarrow+\infty .
$$

Arguing as in the proof of Theorem 3.2 iv (b) we obtain the existence of a one-parameter family of solutions. The behavior of the solutions near $\xi=0$ follows at once from the relations

$$
p-I=h^{\frac{1}{\beta+\gamma}} \approx e^{\frac{\rho y}{\beta+\gamma}} \psi^{* \frac{1}{\beta+\gamma}}=\omega^{\frac{1-\alpha}{\gamma+1}}\left(\frac{\lambda^{2} I(\gamma+1)}{a(1-\alpha)}\right)^{\frac{1}{\gamma+1}}, \quad \frac{\mathrm{d} p}{\mathrm{~d} \xi} \approx-\lambda I \omega^{-\alpha} .
$$

Proof of (iv). We proceed as in the proof of iii) and introduce $\varphi=e^{-r y} u$ and $\psi=e^{-\rho y} h$. This yields the system

$$
\left\{\begin{array}{l}
\frac{\mathrm{d} \varphi}{\mathrm{d} y}=-(r+1) \varphi+e^{-r y}\left(-\frac{\varepsilon^{2} a}{\lambda^{2}}\left(1-e^{y}\right) \frac{\psi}{\varphi} e^{(\mu-1+\rho-r) y}\right) \\
\frac{\mathrm{d} \psi}{\mathrm{d} y}=-\rho \psi+e^{\frac{\rho}{\beta+\gamma} y}(\beta+\gamma) \frac{\varepsilon^{2}}{\varphi} \psi^{\frac{2 \beta+\gamma}{\beta+\gamma}} e^{\left(\mu-\alpha+\rho \frac{\beta}{\beta+\gamma}-r\right) y}
\end{array}\right.
$$

Requiring that $\mu-1+\rho-r=\mu-\alpha+\rho \frac{\beta}{\beta+\gamma}-r=0$ we obtain

$$
\rho=\frac{(1-\alpha)(\beta+\gamma)}{\gamma} \quad \text { and } \quad r=\mu-1+\frac{(1-\alpha)(\beta+\gamma)}{\gamma} .
$$

By 23 both $\rho$ and $r$ are positive. We set $t=e^{-r y}$. Then

$$
\left\{\begin{array}{l}
\frac{\mathrm{d} \varphi}{\mathrm{d} t}=\frac{(r+1)}{r t} \varphi-\frac{1}{r}\left(-\frac{\varepsilon^{2} a}{\lambda^{2}}\left(1-t^{-1 / r}\right) \frac{\psi}{\varphi}\right), \\
\frac{\mathrm{d} \psi}{\mathrm{d} t}=\frac{\psi}{r t}\left(\rho-(\beta+\gamma) \frac{\varepsilon^{2}}{\varphi} \psi^{\frac{\beta}{\gamma+\beta}}\right),
\end{array}\right.
$$


and arguing as before it is natural to look for solutions converging, as $\rightarrow \infty$, to $\left(\varphi^{*}, \psi^{*}\right)$, where

$$
\varphi^{*}=\frac{\varepsilon^{2} a}{\lambda^{2}} \psi^{*} \quad \text { and } \quad \psi^{*}=\left(\frac{\lambda^{2} \gamma}{a(1-\alpha)}\right)^{1+\beta / \gamma} .
$$

Linearizing around $\left(\varphi^{*}, \psi^{*}\right)$ yields the matrix

$$
\left(\begin{array}{cc}
-\frac{1}{r \varphi^{*}} & \frac{\varepsilon^{2} a}{\lambda^{2} r \varphi^{*}} \\
\frac{\lambda^{2}(1-\alpha)(\beta+\gamma)}{\varepsilon^{2} a \gamma r t} & -\frac{\beta(1-\alpha)}{\gamma r t}
\end{array}\right),
$$

and introducing $s$ as before we find a smooth center manifold orthogonal, at $\left(\varphi^{*}, \psi^{*}, 0\right)$, to $\left(-\lambda^{2}, \varepsilon^{2} a, 0\right)$. The equations for $\psi(\tau)-\psi^{*}$ and $s(\tau)(\tau=\log t)$ on the center manifold are, in linear approximation, governed by

$$
\left(\begin{array}{cc}
\frac{1-\alpha}{r} & 0 \\
0 & -\frac{1}{n}
\end{array}\right) .
$$

and the rest of the proof is identical to the previous one.

\section{Acknowledgments}

The authors thank G. I. Barenblatt for having suggested the problem and many stimulating discussions. They thank B. H. Gilding for providing the correct references for the material of Section 2 and S. Bianchini for a long discussion about the proof of Theorem 3.2 ivp (b); its present version is based on his suggestions and has replaced an earlier, rather ad hoc, proof. The second author expresses his deep gratitude for the hospitality of the Lawrence Berkeley National Laboratory, where most of this paper was prepared. This work was supported in part by the Office of Science, Office of Advanced Scientific Computing Research, Mathematical, Information, and Computational Sciences Division, Applied Mathematical Sciences Subprogram, of the U.S. Department of Energy, under Contract No. DE-AC03-76SF00098.

\section{REFERENCES}

1. Aronson, D. G. The porous medium equation. Nonlinear Diffusion Problems, A. Fasano and M. Primicerio (eds.), Lecture Notes in Math. 1224, Springer (1986), 1-46. Zbl 0626.76097 MR 0877986

2. Barenblatt, G. I., Bertsch, M., \& Nitsch, C. Nonlocal damage accumulation and fluid flow in diatomites. Comm. Appl. Math. Comput. Sci. 1 (2006), 143-168. Zbl 1102.76070 MR 2244273

3. Barenblatt, G. I., Entov, V. M., \& Ryzhik, V. M. Theory of Fluid Flows Through Natural Rocks. Kluwer (1990). Zbl 0769.76001

4. Barenblatt, G. I., Patzek, T. W., Prostokishin, V. M., \& Silin, D. B. SPE 75230: Oil deposit in diatomites: A new challenge for subterranean mechanics. SPE/DOE Improved Oil Recovery Symposium (2002).

5. Barenblatt, G. I., \& Prostokishin, V. M. A mathematical model of damage accumulation taking into account microstructural effects. Eur. J. Appl. Math. 4 (1993), 225-240. Zbl 0789.73053 MR 1236341 
6. Bertsch, M., DAl PAsso, R., \& Nitsch, C. A system of degenerate parabolic nonlinear PDE's: a new free boundary problem. Interfaces Free Bound. 7 (2005), 255-276. Zbl 1079.35102 MR 2171132

7. CARr, J. Applications of Centre Manifold Theory. Appl. Math. Sci. 35, Springer (1981). Zbl 0464.58001 MR 0635782

8. Gilding, B. H., \& Kersner, R. Travelling Waves in Nonlinear Diffusion-Convection Reaction. Birkhäuser (2004). Zbl 1073.35002 MR 2081104

9. Hale, J. K. Ordinary Differential Equations. Pure Appl. Math. 21, Wiley-Interscience, New York (1969). Zbl 0186.40901 MR 0419901

10. KAтоK, A., \& Hasselblatt, B. Introduction to the Modern Theory of Dynamical Systems. Encyclopedia Math. Appl. 54, Cambridge Univ. Press (1995). Zbl 0878.58020 MR 1326374

11. Nitsch, C. Some mathematical problems in nonlocal continuum damage mechanics. Ph.D. thesis, Università di Roma "La Sapienza" (2003); http://wpage.unina.it/c.nitsch/PhdThesis.ps

12. VÁzquez, J. L. The Porous Medium Equation. Mathematical Theory. Oxford Math. Monogr., Oxford Univ. Press (2007). Zbl 1107.35003 MR 2286292 